\title{
El hospital como dinamizador y difusor de salud regional: la experiencia del Hospital de Tacuarembó, Uruguay
}

Hospitals as a way to dynamize and disemminate regional health: the case of the Hospital de Tacuarembó, Uruguay

0 hospital como habilitador e difusor da saúde regional: a experiência do hospital de Tacuarembó, Uruguai

Jorge A. Guisantes', Ciro Ferreira², Sebastián Segura³, Jacqueline Gómez², Gustavo Pereyra², Antonio Turnes ${ }^{4}$

\section{Resumen}

Se describe el desarrollo del Hospital Regional de Tacuarembó (HRT), situado en una ciudad mediana del norte de Uruguay, que lo condujo a convertirse en un referente dinamizador de la salud regional, con una atención médica moderna y eficaz, así como de servicio a la comunidad y a la salud nacional. Todo ello logrado a través de una gestión ambiciosa y optimista en los objetivos, basada en esfuerzos colectivos e individuales así como en apoyos institucionales nacionales y de organismos internacionales. Ese papel dinamizador e influyente a nivel regional, e inclusive nacional, está basado, por una parte, en una gran mejora de su base logística, es decir, el propio hospital, en cuanto a instalaciones, equipamientos y ampliación de las capacidades de atención médica; pero también en un gran apoyo e impulso a la Atención Primaria en Salud, no solo en la ciudad de Tacuarembó, sino a nivel departamental. Además, el HRT ha jugado un papel importante en el control de dos zoonosis parasitarias de importancia nacional: la enfermedad de Chagas y la equinococosis quística. Esa acción dinamizadora ha consistido en plantear al hospital como una "organización de salud", considerando el contexto de un territorio particular junto a los determinantes del sistema nacional de salud. Se puede considerar al HRT como una organización innovadora liderada por "emprendedores sociales" dentro de un marco de emprendimiento social partiendo de un hospital departamental o provincial.

Palabras clave: Hospitales

Atención primaria de salud

Key words: Hospitals

Salud pública

Primary health care

Tacuarembó

Public health

Zoonosis

Tacuarembó

Zoonoses

\footnotetext{
1. Universidad del País Vasco, Vitoria, España.

2. Hospital de Tacuarembó, Tacuarembó, Uruguay.

3. SISTAC, Hospital de Tacuarembó, Tacuarembó, Uruguay.

4. Academia Nacional de Medicina de Uruguay, Montevideo, Uruguay.

Correspondencia: Jorge A. Guisantes. Depto. de Inmunología, Microbiología y Parasitología, Universidad del País Vasco, Paseo de la Universi-

dad 7, Vitoria, España. Correo electrónico: jorgegdelb@gmail.com

Los autores declaran no tener conflictos de intereses.

Recibido: 28/5/20

Aprobado: 21/10/20

Attribution-NonCommercial 4.0 International (CC BY-NC 4.0)
} 


\section{Introducción}

La evolución del concepto de hospital ha ido cambiando a través de los siglos. Desde los antiguos hoteles o albergues de peregrinos y los asilos de enfermos, a los más completos e inclusive especializados del siglo XIX y comienzos del siglo XX, pasando a una actividad más amplia en el cuidado de la salud, más compleja, más especializada y de más alto nivel, tal como señala $\mathrm{A}$. Turnes en su revisión sobre la evolución histórica de los hospitales en diferentes países y culturas desde el siglo IV $\mathrm{aC}^{(1)}$.

A partir de la Declaración de Alma Ata, Kazajistán, en 1978, en la conferencia internacional organizada por la Organización Mundial de la Salud (OMS) y la Organización de las Naciones Unidas para la Educación, la Ciencia y la Cultura (UNICEF) ${ }^{(2)}$, se le ha ido dando importancia creciente a la Atención Primaria de Salud (APS), a la vez que su relación con la atención hospitalaria fue cobrando fuerza. Es así como surge o se va formando el concepto del hospital como dinamizador y defensor de la salud pública en una región, con su labor asociada y colaboradora de la $\operatorname{APS}^{(3-6)}$.

Un claro ejemplo de cómo un hospital regional puede convertirse en un dinamizador y promotor de la salud no solo en su ciudad, sino también en una región, lo tenemos en un hospital de Sudamérica: el Hospital Regional de Tacuarembó (HRT), situado en la ciudad y departamento del mismo nombre de la República Oriental del Uruguay.

Por lo tanto, será objetivo de esta comunicación describir en forma resumida cómo en el transcurso de 25 años, un pequeño hospital departamental de una ciudad mediana del norte de Uruguay pasó a convertirse en un referente regional y nacional de atención médica moderna y eficaz, así como de servicio a la comunidad y a la salud nacional.

Todo ello logrado a través de una gestión ambiciosa y optimista en los objetivos, basada en esfuerzos colectivos e individuales, así como en apoyos institucionales nacionales y de organismos internacionales.

\section{La base logística: el hospital}

Uruguay tiene una superficie terrestre de $175.016 \mathrm{~km}^{2}$ con una población total estimada para 2017 de 3.493.205 habitantes ${ }^{(7)}$. Está dividido administrativa y políticamente en 19 departamentos (figuras 1 y 2). El departamento de Tacuarembó, el mayor del país, tiene una superficie de $15.438 \mathrm{~km}^{2}$ y una población estimada de 93.039 habitantes, de los cuales 71.772 viven en su capital, Tacuarembó ${ }^{(7)}$, donde está situado el HRT. Los departamentos limítrofes de Artigas, Rivera, Salto y Paysandú (Región Norte del río Negro) tienen una

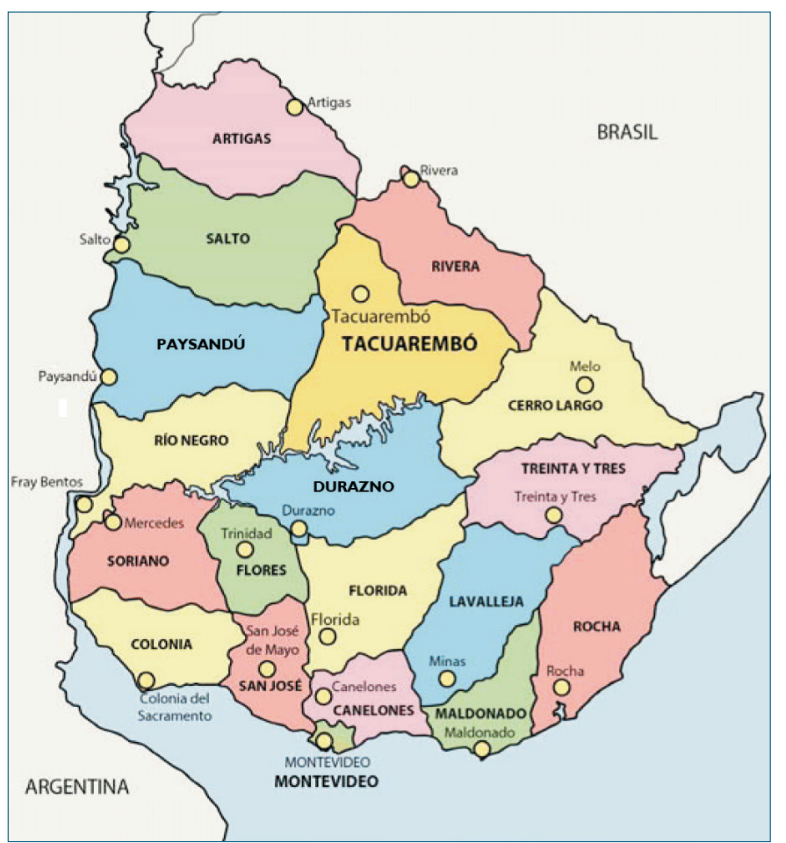

Figura 1. Ubicación geográfica y división político administrativa de la República Oriental del Uruguay.

población conjunta de 527.556 habitantes en 64.821 $\mathrm{km}^{2}$. Si añadimos el departamento de Cerro Largo, área de influencia del HRT, la población total vinculada de alguna forma al HRT sería de 617.113 habitantes en $78.469 \mathrm{~km}^{2(7)}$.

El Hospital de Tacuarembó del Ministerio de Salud Pública (MSP) fue inaugurado el 29 de setiembre de 1927 por la Asistencia Pública Nacional (APN). La APN fue una organización del Estado, creada por Ley No. 3.724 del 7 de noviembre de 1910, que dirigió los hospitales públicos desde 1911 hasta 1932, pasando sus atribuciones al Consejo de Salud Pública (que reunió las funciones de asistencia y de higiene) hasta el 12 de enero de 1934 en que fue promulgada la Ley No. 9.202 creadora del $\mathrm{MSP}^{(8,9)}$, pasando en 1953 a denominarse Centro Departamental de Salud Pública de Tacuarembó (Hospital Regional). Actualmente depende de la Administración de los Servicios de Salud del Estado (ASSE). En los últimos años ha experimentado un importante crecimiento pasando de tener una planta física de $6.000 \mathrm{~m}^{2}$ en 2005 a tener casi $11.000 \mathrm{~m}^{2}$ en 2017, aumento que se ha distribuido fundamentalmente en cuatro grandes áreas: Área de Cuidados Críticos (incluyendo Cuidados Intensivos de Adultos, Cuidados Intensivos Neonatales y Servicio de Cirugía), Área del Centro de Imagen, Área del Centro Oncológico y Área Ambulatoria ${ }^{(10,11)}$.

El HRT es un hospital del tercer nivel de atención, con servicios regionales especializados que actualmente abarcan también a otros departamentos al norte del río Negro. Según Gómez ${ }^{(12)}$, la población asistida por el sis- 


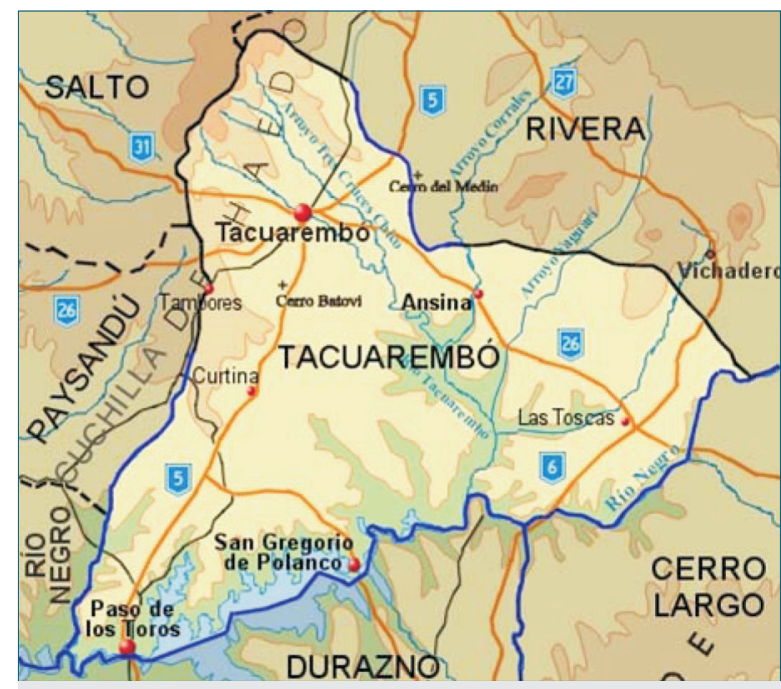

Figura 2. Departamento de Tacuarembó, República Oriental del Uruguay.

tema público de salud en el departamento de Tacuarembó es el $64 \%$ de la población total. Por lo tanto, y de acuerdo a esas estimaciones, esto significaría que al ser un hospital regional, el HRT atiende a unos 59.545 usuarios departamentales propios y a unos 337.636 usuarios de la Región Norte ${ }^{(7)}$. Si incluimos en su área de influencia al departamento de Cerro Largo, ese 64\% implica una población total de influencia en atención pública de salud de 394.952 habitantes.

El HRT brinda los siguientes servicios a la población: Urgencias, Cirugía, Medicina Interna, Pediatría, Obstetricia y Ginecología, Unidad de Cuidados Intensivos (UCI) Neonatales, UCI de Adultos, UCI Intermedios, Centro de Imagen, Farmacia Hospitalaria, Centro de Oncología, Centro de Neurocirugía, Banco de Leche Humana, Banco de Sangre, Laboratorio Central, Policlínicas de Otorrinolaringología, Psiquiatría, Odontología y Cardiología ${ }^{(8)}$. Cuenta también con el Centro de Salud Departamental donde actualmente tiene su sede la Red de Atención Primaria (RAP).

En la evolución y mejora de los servicios del HRT ha sido un hito la creación de un Banco de Leche Humana (BLH), en setiembre de 2004, que será el primero en el interior del país. El BLH dispone de un equipo automatizado de pasteurización que da alta seguridad en el manejo de la leche materna obtenida de madres donantes sanas con hijos vivos. Este banco ha sido de gran importancia para el cuidado de los recién nacidos prematuros, aportando todas las ventajas conocidas de la lactancia materna en cuanto a desarrollo inmunitario y nutrición.

Otro hito importante alcanzado ha sido la creación y puesta en marcha en setiembre de 2001 del Centro Regional de Neurocirugía de Tacuarembó (CERENET), brindando asistencia neuroquirúrgica a los hospitales de la Región Norte del río Negro y al departamento de Cerro Largo ${ }^{(13)}$. Actualmente realiza unas 30 neurocirugías al mes. Atiende tanto a pacientes públicos como privados, contando con un equipo médico y de enfermería altamente capacitado y con tecnología puntera, incluyendo un angiógrafo de última generación.

Es también digna de señalar por su importancia regional la puesta en marcha en 2009 del Centro Oncológico del Norte, que da un servicio integral a los pacientes oncológicos en cuanto a diagnóstico, tratamiento y seguimiento, y que está dotado del equipamiento más actualizado en materia de tomografía y radioterapia ${ }^{(8)}$, incluyendo un Área de Hospital de Día para los tratamientos ambulatorios. Este centro, teniendo en cuenta la población subsidiaria del HRT, tendrá al menos 350 pacientes nuevos tratados por año.

En el año 2008 se crea un Centro de Rehabilitación con ayuda de la comunidad. Dicho centro, cercano al HRT, recibe pacientes de las policlínicas rurales dirigidos al hospital.

\section{El hospital y la Atención Primaria de Salud}

Cabe recordar, en relación con la APS, que Uruguay fue uno de los países que suscribieron la Declaración Internacional sobre APS de Alma-Ata en $1978^{(2)}$. La relación entre el HRT y la APS comienza en 1985 con la iniciativa denominada "Proyecto Tacuarembó", que propuso coordinar una acción conjunta entre la División Central de APS del MSP, la Intendencia Departamental de Tacuarembó (IDT) y el HRT, con la firme decisión de que el hospital saliera de su edificio, implicándose en la salud de la comunidad regional. Posteriormente, otras instituciones se fueron involucrando en el proyecto, tales como el Sindicato Médico de Tacuarembó, el Ministerio del Interior, la Administración Nacional de Educación Pública (ANEP) y otras. Pero será a partir de 1991 que se ejecutará el Proyecto "Fortalecimiento de la APS en Tacuarembó, Uruguay" con el apoyo económico y la asesoría de la Agencia Alemana de Cooperación Técnica (Deutsche Gesellschaft für Technische Zusammenarbeit - GTZ) coordinada por el Dr. Hermes Vallejo, consultor de dicha agencia ${ }^{(14)}$.

Para poder llevar a cabo la estrategia multisectorial y multifactorial necesaria, se creó una Oficina de Desarrollo de la Atención Primaria de Salud (Oficina DAPS) en el propio HRT. Tal como comentan Vallejo y López-Ramos $^{(14)}$, es muy probable que los avances logrados en la implementación de la APS en Tacuarembó no hubieran sido posibles sin esa oficina ejecutora, integrada totalmente por personal de contrapartida nacional, y desde donde se condujo el proyecto y la asesoría de GTZ. La Oficina DAPS fue una estructura de coordinación inter- 
sectorial sustentada también por el MSP y la IMT, que aportaron personal, dinero y materiales para su mantenimiento y operación. Las metas claras establecidas por la Oficina DAPS, el compromiso de sus participantes y la flexibilidad en el trabajo, contribuyeron al buen funcionamiento y al logro de los objetivos planteados ${ }^{(14)}$.

La cooperación entre la Oficina DAPS del HRT y la agencia alemana GTZ significó importantes aportes en cuanto a la capacitación del personal de salud, el mejoramiento de la salud rural, incluyendo policlínicas y puestos de salud rurales, conectados con una base de radio instalada en el HRT, el apoyo a los planes nacionales de control de la enfermedad de Chagas y de la equinococosis quística, la participación comunitaria en los programas de salud, la creación del Sistema de Información en Salud del Departamento de Tacuarembó (SISTAC) y el fortalecimiento de la APS en Tacuarembó, incluyendo el asesoramiento a los departamentos limítrofes de Durazno y Rivera. El HRT actuó de esta manera como un dinamizador de la APS regional. También se definieron varios Programas Prioritarios de la Salud, tales como: i) Promoción de la lactancia materna, ii) la creación de una Clínica para adolescentes; iii) Enfermedades crónicas del adulto; iv) Programa de prevención del cáncer genital femenino; v) Programa de salud bucal ${ }^{(13)}$.

La experiencia de la Oficina DAPS sirvió como germen para la RAP dentro de la recomendación nacional hacia una descentralización de la atención médica a nivel regional, facilitando una gestión independiente de la APS. La RAP que tiene su sede departamental en el HRT, desde el punto de vista operativo sigue teniendo una importante vinculación con el HRT. Éste, entre otros aspectos que se comentarán más adelante, lleva a que Tacuarembó siga estando en la avanzada de la descentralización de la atención médica.

\section{Papel del HRT en el control de la enfermedad de Chagas en Tacuarembó}

El HRT colaboró activamente en la lucha contra la enfermedad de Chagas en Tacuarembó. Cabe señalar que, según las investigaciones realizadas por el Programa Nacional de Chagas del MSP, con el apoyo de la Organización Panamericana de la Salud (OPS), en el año 1986, Tacuarembó era uno de los departamentos con mayor endemia chagásica, con una tasa de prevalencia humana de $6 \%{ }^{(15,16)}$. En 1988, el HRT crea la Policlínica de Enfermedad de Chagas, adonde se derivaban los casos positivos en estudio, apoyando así la lucha contra la zoonosis. En los aspectos prácticos, el HRT fue la sede operativa donde se centralizaban las acciones del Plan Nacional de Chagas para ese departamento. Asimismo, en el laboratorio clínico del HRT se realizaron estudios serológicos para la determinación de la prevalencia de la infección chagásica en la población departamental. Diferentes encuestas serológicas realizadas en los años 1988, 1990, 1992, 1995 y 1998 permitieron seguir la evolución favorable de la lucha contra la enfermedad de Chagas, que descendió a tasas de prevalencia humana de $1 \%{ }^{(17)}$. A su vez, los índices de infestación domiciliaria por triatomíneos bajaron de tasas de $16 \%$ en 1985 a $0,7 \%$ en 1996 , como resultado del combate domiciliario al vector transmisor, al que se añadió un plan de mejoramiento de la vivienda rural insalubre.

En 1997 se certificó la interrupción de la transmisión vectorial domiciliaria de la enfermedad de Chagas en Uruguay y en 2013 la eliminación de Triatoma infestans como problema de salud pública ${ }^{(18)}$.

\section{Papel del HRT en los programas de control de la hidatidosis en Uruguay}

La equinococosis quística o hidatidosis, producida por Echinococcus granulosus, es una antropozoonosis endémica en la región. El HRT colaboró en los planes de la Comisión Nacional de Zoonosis del MSP para el control de esta zoonosis, uniendo sus recursos a los de la Oficina DAPS y contando, a su vez, con el apoyo de la Oficina en Uruguay de la OPS/OMS, así como del asesoramiento internacional. Como una medida de cooperación con el Programa Nacional de Control de la Hidatidosis, el HRT creó en su estructura la Policlínica de Hidatidosis, especializada y exclusiva, que funciona en segundo nivel hospitalario, en la cual trabajan distintos especialistas: ecografistas, cirujanos, médicos internistas, laboratoristas, enfermeras, asistentes sociales y educadores para la salud, junto al personal administrativo correspondiente ${ }^{(14)}$. Esta policlínica está orientada a dar confirmación diagnóstica, tratamiento y seguimiento clínico a los casos provenientes del área rural que son sospechosos de ser portadores de quistes hidatídicos. Son casos detectados mediante estudios de ecografía de masas y estudios serológicos realizados de acuerdo con el Programa Nacional de Control de la Hidatidosis ${ }^{(19)}$. Por lo tanto, el HRT ha sido y es un importante apoyo en la imprescindible orientación de los pacientes del departamento a los efectos de instaurar cuanto antes el tratamiento médico o quirúrgico que corresponda, así como el seguimiento posterior. Todo ello encuadrado en el Programa Nacional de Control de la Hidatido$\operatorname{sis}^{(19)}$. Ello condujo, en una tarea de muchos años, a un mejor conocimiento de la endemia humana en la región, lo cual, posteriormente, derivó en una menor incidencia humana y, sobre todo, en un mejor seguimiento médico y epidemiológico de los pacientes. 


\section{El hospital y la salud rural}

El HRT también ha jugado un papel importante de apoyo a las acciones llevadas a cabo por la Oficina DAPS mediante el Programa de Rondas Médicas Rurales, creado para brindar accesibilidad y, por lo tanto, mejorar la cobertura sanitaria de la población del área rural dispersa del departamento, calculada en aquellos años en un $18 \%{ }^{(14)}$. Para conducir ese programa se dividió el área rural en 12 zonas donde desarrollaron sus actividades los denominados médicos de ronda y rurales, quienes con diferente frecuencia (semanal, quincenal o mensual), visitaban a la población de dichas zonas. Para facilitar su acción se crearon diferentes puntos de atención denominados, según su importancia, Puestos de Salud, Policlínica General y Policlínica Integral. La actividad desarrollada fue importante como indica, por ejemplo, que entre los años 1990 y 1997 se realizó una media anual de 21.774 consultas rurales y rondas médi$\operatorname{cas}^{(14)}$.

Para sus actividades, la Oficina DAPS siempre contó con el respaldo del HRT, que con sus estructuras y servicios daba seguridad al sistema de atención médica rural. Para ello, entre otras acciones, se creó en el HRT una base de radio con móviles en zonas estratégicas rurales que no contaban con otros servicios de telecomunicaciones. Asimismo, desde el HRT se instrumentó un sistema de comunicación de los médicos rurales y de ronda con el laboratorio clínico hospitalario, para tener una mayor disponibilidad horaria y contar con una comunicación permanente frente a situaciones clínicas concretas. También desde el HRT se logró coordinar el traslado de pacientes de zonas rurales para ser atendidos por especialistas del hospital $^{(14)}$. Por lo tanto, es evidente el importante papel jugado por el HRT en apoyo a la APS de las zonas rurales del departamento, con las evidentes mejoras para la salud comunitaria.

\section{Innovación y mejora mediante la descentralización}

El desarrollo de la atención sanitaria en Uruguay ha girado siempre en torno a la capital del país, Montevideo, en donde se han localizado los principales centros hospitalarios de tercer nivel de la red de ASSE y en donde se ejerce la docencia universitaria de medicina, enfermería y ramas afines. También en Montevideo se localiza el Hospital de Clínicas "Dr. Manuel Quintela", dependiente de la Universidad de la República.

Cabe señalar que ASSE, que fue creada como organismo desconcentrado dependiente del MSP por Ley No. 15.903 de 10 de noviembre de 1987 (artículos 267 a 282), y transformada en 2007, por Ley No. 18.161 de 29 de julio de 2007, denominada "Creación de la Administración de los Servicios de Salud del Estado (ASSE) co- mo servicio descentralizado", se define oficialmente como el principal prestador estatal de atención integral de salud en todo el territorio nacional, encargándose a través de la red de hospitales del país de brindar servicios de atención de salud integral, incluyendo prevención, diagnóstico precoz, tratamiento, rehabilitación y cuidados paliativos. Esto, lógicamente, añade complejidad a la tarea, pero que ha sido resuelta muy bien por el grupo directivo del HRT.

La centralización en la atención sanitaria había conducido a que una parte importante de la población uruguaya, para resolver sus problemas médicos tanto de diagnóstico como de tratamiento, debía desplazarse a la capital desde las ciudades y pueblos del interior del país. Esto conllevaba a una diferencia en el trato sanitario claramente desventajosa para casi la mitad de la población $^{(20)}$, con claros perjuicios para la calidad y rapidez del diagnóstico y tratamiento. Debido a eso, todos los intentos innovadores para solucionar esa discriminación negativa se señalan como un ejemplo a seguir.

En su libro, Villar y Ferreira ${ }^{(20)}$ describen muy bien las ventajas de la descentralización de la atención sanitaria, dándole un mayor papel a los hospitales regionales. Pero para ello es necesario dotarlos de los equipamientos y del personal cualificado para poder llevarlo a cabo, así como asegurar la financiación para su adecuado y continuo funcionamiento.

$\mathrm{Al}$ respecto, podemos afirmar que el HRT es pionero en esta descentralización e innovación de la atención médica en Uruguay. En el excelente informe de Sutz y colaboradores $^{(21)}$ se analiza muy bien la innovación tecnológica y social en salud pública que ha supuesto la actividad del HRT, actuando como un foco dinamizador de una mejor y más descentralizada atención en salud. Esa acción dinamizadora consiste en plantear al hospital como una "organización en salud", considerando el contexto de un territorio particular junto a los determinantes del sistema nacional de salud. Hay que tener en cuenta que en la esfera de la salud la mayoría de las desigualdades conducen a situaciones de exclusión social ${ }^{(21)}$.

La innovación local y regional en salud desarrollada por el HRT se ha efectuado institucional y políticamente dentro del SNIS del MSP, que determina los objetivos y las responsabilidades de cada organización ${ }^{(21)}$. El SNIS está regulado por la Ley 18.211 de 5/12/2007 con las modificaciones y los agregados efectuados por las leyes 18.731 y 18.732 de $7 / 1 / 2011$. Según el enunciado de dicha ley, el SNIS asegurará el acceso a servicios integrales de salud a todos los habitantes residentes en el país, promoviendo la salud con énfasis en los factores determinantes del entorno y los estilos de vida de la población. Y entre sus objetivos el enunciado de la ley señala 
que se intenta "Alcanzar el más alto nivel posible de salud de la población mediante el desarrollo integrado de actividades dirigidas a las personas y al medio ambiente que promuevan hábitos saludables de vida, y la participación en todas aquellas que contribuyan al mejoramiento de la calidad de vida de la población". Y muy particularmente: "Impulsar la descentralización de la ejecución en el marco de la centralización normativa, promoviendo la coordinación entre dependencias nacionales y departamentales". Objetivo de descentralización que ha estado en la base de la actividad del HRT, como señalamos más arriba.

Tres actividades resaltan como importantes en la descentralización innovadora llevada a cabo por el HRT: el Banco de Leche Humana, el Centro de Neurocirugía y el Centro Oncológico. Ambos centros son referencia y punto de atención para el territorio uruguayo al norte del río Negro, el cual abarca una superficie de $64.821 \mathrm{~km}^{2}$ y una población de 527.556 habitantes. Si, como se señaló anteriormente, se incluye a Cerro Largo en el área de influencia sanitaria del HRT, la población total asciende a 617.113 habitantes $^{(7)}$.

Cabe señalar que la experiencia innovadora del HRT está basada no en un hecho o acto puntual en el tiempo, sino que es el resultado de un proceso continuo de innovación y mejora iniciado en 1992 y que continúa en la actualidad con nuevas adquisiciones de equipos (como un angiógrafo de última generación ya operativo) destinados a la creación de un centro de alta tecnología cardiovascular.

Tal como indican Sultz y colaboradores ${ }^{(21)}$, el HRT es una organización innovadora liderada por "emprendedores sociales". Esa evolución no ha sido algo aleatorio, sino que ha seguido una trayectoria marcada por un estilo apropiado de dirección desarrollado por el equipo directivo del HRT. El HRT no es un centro de investigación ni de alta tecnología, aunque tiene equipamientos que sí lo son, sino que es un hospital que ha buscado soluciones apropiadas para problemas de atención médica locales y regionales basándose en una dinámica de innovación propia, que es una mezcla de mejoría de la gestión e innovación tecnológica ${ }^{(21,22)}$. Es lo que se podría definir como una actividad de emprendimiento social ${ }^{(23)}$. Según Dees, hay tres características básicas de las actividades de emprendimiento social: i) el reconocimiento y aprovechamiento de nuevas oportunidades de servir en una misión de impacto social; ii) involucrarse en un proceso de innovación; iii) actuar sin estar limitado por los recursos disponibles en ese momento. A ellas se podría añadir, según Peredo y McLean, la tolerancia al riesgo asumido ${ }^{(24)}$. El HRT reúne todas estas características de emprendimiento social $^{(21)}$.
Esta actividad descentralizadora e innovadora del HRT ha sido reconocida y avalada en diferentes publicaciones científicas ${ }^{(25-27)}$ y premios internacionales obtenidos, tales como la nominación Hospital Amigo del Niño por la UNICEF en 1997 y el Premio de la Federación Internacional de Ginecología y Obstetricia (FIGO) en $2003^{(8)}$

Por otra parte, la actividad y el prestigio del HRT han sido reconocidas por la población, que en diferentes encuestas de opinión pública realizadas por Equipos Mori Consultores Asociados, ha valorado muy positivamente la calidad de los servicios prestados aceptando muy bien el hecho de que el HRT atienda pacientes de fuera del departamento, así como la cooperación entre la salud pública, el sector privado y la $\operatorname{IDT}^{(8)}$.

\section{Conclusiones}

La actividad de atención médica extendida a una región desde un hospital de una ciudad mediana, su labor de apoyo y desarrollo de la atención primaria en salud a nivel regional, el desarrollo de especialidades de diagnóstico y tratamiento médico que son referencia regional y nacional, el papel importante que tiene en salud neonatal e infantil, su papel en el control de importantes enfermedades zoonóticas en su región, su apoyo a programas de salud rural, el impacto que ha tenido en la mejora de la situación social de su departamento y el reconocimiento de su labor por parte de la población, así como por organismos internacionales, merecen que la experiencia del HRT sea conocida a nivel nacional e internacional como ejemplo de lo que puede hacerse dentro de un marco de emprendimiento social partiendo de un hospital departamental o provincial.

\section{Agradecimientos}

Los autores agradecen a Marina E. Guisantes su colaboración en las figuras del presente artículo.

\section{Summary}

The study presents the case of the Hospital de Tacuarembó (HRT), located in a medium-size city in the north of Uruguay, which turned into a reference in terms of the dynamization of regional health, offering modern and effective healthcare services, serving the community and contributing to the improvement of national health.

All of this was possible as a result of an ambitious and optimistic management of objectives, based on collective and individual effort, as well as relying on the support of national and international bodies. This dynamizing and influential role played by the Hospital de Tacuarembó is based, on the one hand on a great improvement of its logistical base - that is, the hospital itself - in 
terms of its premises, equipment and upscaling of medical services, and on the other hand on the major support and stimulus to Primary Healthcare Services, not only in the city of Tacuarembó, but also throughout the entire province. Likewise, the HRT has played a significant role in the control of two parasite zoonoses that are extremely important for Uruguay: Chagas disease and cystic echinococcosis. This dynamizing actions have consisted in regarding the hospital as a "health organization" within the context of a certain territory, along with factors defined by the national health system. HRT may be considered as an innovative organization led by "social entrepreneurs" within the framework of social entrepreneurship, stemming from a departmental or province hospital.

\section{Resumo}

Descreve-se o desenvolvimento do Hospital Regional de Tacuarembó (HRT), localizado em uma cidade de médio porte do norte do Uruguai, o que o tornou uma referência dinâmica de saúde regional, com atendimento médico moderno e eficaz, além de atendimento à comunidade e a saúde nacional. Tudo isto alcançado através de uma gestão ambiciosa e otimista dos objectivos, baseada no esforço coletivo e individual, bem como no apoio institucional nacional e de organismos internacionais. Este papel dinâmico e influente a nível regional, e mesmo nacional, está baseado, por um lado, numa grande melhoria da sua base logística, ou seja, do próprio hospital, ao nível das instalações, equipamentos e ampliação das capacidades de assistência médica; mas também num grande apoio e dinamização da Atenção Primária à Saúde (APS) não só na cidade de Tacuarembó mas também a nível departamental. Além disso, o HRT tem desempenhado um papel importante no controle de duas zoonoses parasitárias de importância nacional: a doença de Chagas e a equinococose cística. Essa dinâmica de ação consistiu em considerar o hospital como uma "organização de saúde", considerando o contexto de um determinado território e os determinantes do sistema nacional de saúde. O HRT pode ser considerado uma organização inovadora liderada por "empreendedores sociais" dentro de um quadro de empreendedorismo social a partir de um hospital departamental ou provincial.

\section{Bibliografía}

1. Turnes A. Historia y evolución de los hospitales en las diferentes culturas. Montevideo: SMU, 2009. En: https://www. smu.org.uy/dpmc/hmed/historia/articulos/origen-y-evolucion.pdf. [Consulta: 4 abril 2019].

2. Organización Mundial de la Salud. Atención Primaria de Salud. Alma-Ata. Informe sobre la Conferencia Internacional sobre Atención Primaria de Salud. Alma-Ata, URSS, 6-12 septiembre 1978. Ginebra. OMS, 1978. Disponible en: https://apps.who.int/iris/bitstream/handle/10665/39244/9243541358.pdf? sequence $=1 \&$ isAllowed $=y$. [Consulta: 4 abril 2019].

3. Organización Mundial de la Salud. Los hospitales y la salud para todos: informe de un Comité de Expertos de la OMS sobre la función de los hospitales en el primer nivel de envío de casos. Serie Informes Técnicos 744. Ginebra: OMS, 1987. Disponible en: https://apps.who.int/iris/bitstream/handle/10665/40087/WHO_TRS_744_spa.pdf?sequen$\mathrm{ce}=1$ \&isAllowed $=y$. [Consulta: 4 abril 2019].

4. España. Ministerio de Sanidad y Consumo. La implantación de la promoción de salud en los hospitales: manual y formularios de autoevaluación. Madrid: Ministerio de Sanidad y Consumo, 2007. Disponible en: https://www.mscbs.gob.es/ profesionales/saludPublica/prevPromocion/promocion/hospitalesSalud/docs/PromoSaludHospitales.pdf. [Consulta: 4 abril 2019].

5. Macinko J, Montenegro H, Nebot Adell C, Etienne C, Grupo de Trabajo de Atención Primaria de Salud de la Organización Panamericana de la Salud. La renovación de la atención primaria de salud en las Américas. Rev Panam Salud Publica 2007; 21(2-3):73-84.

6. Vignolo J, Vacarezza M, Álvarez C, Sosa A. Niveles de atención, de prevención y atención primaria de la salud. Arch Med Int 2011; 33(1):11-4.

7. Uruguay. Instituto Nacional de Estadística. Anuario Estadístico 2018. 95 Versión. Montevideo: INE, 2018. Disponible en: https://www.ine.gub.uy/documents/10181/559909/ Anuario+Estad\%C3\%ADstico+Nacional+2018/46660ce3eb26-484e-b295-f4327499de8b. [Consulta: 25 mayo 2019].

8. Turnes A. El Hospital de Tacuarembó: 90 años de su inauguración 1927-2017. Tacuarembó: Hospital de Tacuarembó, 2017.

9. Ferrari J. A 100 años de la ley de la Asistencia Pública Nacional (1910 - 2010), 5 de octubre de 2010. Sesiones de la Sociedad Uruguaya de Historia de la Medicina. Montevideo: Sociedad de Historia de la Medicina, 2010. Disponible en: https://www.smu.org.uy/dpmc/hmed/historia/articulos/100apn.pdf. [Consulta: 4 abril 2019].

10. Trostchansky J. Evolución del Hospital de Tacuarembó en los últimos 20 años. En: Uruguay. Ministerio de Salud Pública. Tacuarembó: horizonte saludable. Montevideo: MSP, 2000:39-41.

11. Martínez L. El hospital y la evolución de su planta física. En: Turnes A. El Hospital de Tacuarembó: 90 años de su inauguración 1927-2017. Tacuarembó: Hospital de Tacuarembó, 2017:247-67.

12. Gómez J. El desafío de llegar al paraje más alejado. Rev ASSE 2014; 1(1):54-7.

13. Villar-Ballestero A. Creación del primer Centro Regional de Neurocirugía de Tacuarembó. En: Villar H, Ferreira C. Descentralización en salud: sistemas departamentales de aten- 
ción. Montevideo: Sindicato Médico del Uruguay, 2005:119-30

14. Vallejo H, López-Ramos O. Fortalecimiento de la Atención Primaria de Salud: Proyecto G.T.Z. - I.M.T. - M.S.P., en el Departamento de Tacuarembó. Montevideo: MSP, Deutsche Gesellschaft für Technische Zusammenarbeit (G.T.Z.), 1999.

15. Salvatella R, Calegari L, Casserone S, Civila E, Carbajal S, Pérez G, et al. Encuesta de prevalencia serológica de la enfermedad de Chagas en Uruguay: informe preliminar parcial (1985). Rev Méd Urug 1986; 2(2):119-25.

16. Salvatella R, Calegari L, Casserone S, Civila E, Carbajal S, Pérez G, et al. Seroprevalencia de anticuerpos contra Trypanosoma cruzi en 13 Departamentos de Uruguay. Bol Ofic Sanit Panam 1989; 107:108-17.

17. Salvatella R, Rosa R, González M, Basmadjian Y, Combol A, Benavides U, et al. Seroprevalencia de la Enfermedad de Chagas en niños de 6 y 12 años en tres departamentos endémicos de Uruguay. Bol Chil Parasitol 1999; 54(3-4):51-6.

18. Salvatella R. Chagas en Uruguay, 1937-2016: información básica para su prevención, control y atención. Arch Pediatr Urug 2016; 87(1):49-52.

19. Irabedra P, Ferreira C, Sayes J, Elola S, Rodríguez M, Morel N, et al. Control programme for cystic echinococcosis in Uruguay. Mem Inst Oswaldo Cruz 2016; 111(6):372-7. doi: 10.1590/0074-02760160070

20. Villar H, Ferreira C. Descentralización en salud: sistemas departamentales de atención. Montevideo: Sindicato Médico del Uruguay, 2005

21. Bianchi C, Bianco M. Research of Innovation Systems and Social Inclusion. Technological and social innovation in health care: the case of the Hospital de Tacuarembó, Uru- guay. RISSI, 2013. Disponible en: http://www.redesist.ie.ufrj.br/rissi-reports/rissi-final-reports. [Consulta: 25 mayo 2019].

22. Salge T, Vera A. Hospital innovativeness and organizational performance: evidence from English public acute care. Health Care Manage Rev 2009; 34(1):54-67. doi: 10.1097/01.HMR.0000342978.84307.80

23. Dees G. The meaning of social entrepreneurship. Durham, NC: Duke University, 2001. Disponible en: https://centers.fuqua.duke.edu/case/wp-content/uploads/sites/7/2015/03/Article_Dees_MeaningofSocialEntrepreneurship_2001.pdf. [Consulta: 26 mayo 2019].

24. Peredo A, McLean M. Social entrepreneurship: a critical review of the concept. J World Bus 2006; 41(1):56-65.

25. Salvatella R. La inserción de un centro asistencial médico dentro de un esquema de atención integral de la Salud. En: Uruguay. Ministerio de Salud Pública. Tacuarembó: horizonte saludable. Montevideo: MSP, 2000:21-3.

26. Guisantes J. Una experiencia dinamizadora de la salud comunitaria. En: Uruguay. Ministerio de Salud Pública. Tacuarembó: horizonte saludable. Montevideo: MSP, 2000:31-2.

27. Bianchi C, Ardanche M, Bianco M, Schenck M. Inclusive innovation and policy mismatch in health care: a Uruguayan local experience. En: Conferência Internacional LALICS 2013 "Sistemas Nacionais de Inovação e Políticas de CTI para um Desenvolvimento Inclusivo e Sustentável". Rio de Janeiro, Brasil 11 e 12 de Novembro, 2013. Disponible en: http://mail.redesist.ie.ufrj.br/lalics/papers/107Inclusive_innovation and policy mismatch in health care A_Uruguayan_local_experience_pdf. [Consulta: 4 abril 2019].

\section{Contribución de autores}

Jorge A. Guisantes, https://orcid.org/0000-0002-5450-949X. Concepción del trabajo, diseño del mismo, conocimiento histórico del asunto, búsquedas bibliográficas, redacción y revisión crítica del manuscrito.

Ciro Ferreira, https://orcid.org/0000-0003-0206-3024. Concepción del trabajo, conocimiento histórico del tema y participación activa en el mismo, aporte de datos y revisión crítica del manuscrito.

Sebastián Segura, https://orcid.org/0000-0002-4858-109X. Aportación de datos y documentos para el estudio, búsquedas en archivos informáticos y manuales del hospital, y revisión crítica del manuscrito.

Jacqueline Gómez, https://orcid.org/0000-0002-4333-2254. Aportación de datos al estudio, participación en el desarrollo del hospital, y revisión del manuscrito.

Gustavo Pereyra, https://orcid.org/0000-0003-3638-0108. Aportación de datos al estudio, participación en el desarrollo del hospital, y revisión del manuscrito.

Antonio Turnes, https://orcid.org/0000-0001-9197-0376. Conocimiento histórico del hospital y del tema como historiador y miembro de la Academia Uruguaya de Historia de la Medicina, aportación de datos de interés y revisión del manuscrito. 